\title{
New approaches to molecular cancer therapeutics
}

\author{
Ian Collins \& Paul Workman
}

\begin{abstract}
Cancer drug development is leading the way in exploiting molecular biological and genetic information to develop 'personalized' medicine. The new paradigm is to develop agents that target the precise molecular pathology driving the progression of individual cancers. Drug developers have benefited from decades of academic cancer research and from investment in genomics, genetics and automation; their success is exemplified by high-profile drugs such as Herceptin (trastuzumab), Gleevec (imatinib), Tarceva (erlotinib) and Avastin (bevacizumab). However, only $5 \%$ of cancer drugs entering clinical trials reach marketing approval. Cancer remains a high unmet medical need, and many potential cancer targets remain undrugged. In this review we assess the status of the discovery and development of small-molecule cancer therapeutics. We show how chemical biology approaches offer techniques for interconnecting elements of the traditional linear progression from gene to drug, thereby providing a basis for increasing speed and success in cancer drug discovery.
\end{abstract}

As in other therapeutic areas, success in innovative small-molecule cancer drug discovery depends on the creative interaction between chemistry and biology. At the heart of the classical drug discovery process are iterative cycles linking chemical synthesis and biological evaluation. Hypotheses generated about therapeutic targets lead to the production of new chemical matter, which is then evaluated in appropriate biological assays. The results generate new hypotheses and stimulate further rounds of synthetic refinement and testing until a compound with the required, predefined properties can be selected for clinical development. Notwithstanding the enormous technical advances made across many fronts, drug discovery remains a rationally driven but essentially empiric process. An important way to improve productivity is to decrease the timescale between hypothesis and feedback. These timescales can be relatively short, as in lead optimization (which may now be completed in as little as 12 to 18 months), to very long, as in clinical trials (which can take many years). In this Review, we show how powerful new technologies can be combined to reduce the cycle times at all stages, so that success or failure is reached faster. It is a concern that despite the increasing number of targeted molecular therapeutics, attrition rates for oncology drugs in the clinic are worse than those for other disease areas $^{1}$ (Box 1). It is therefore important that drugs and molecular targets that will not make it are identified earlier in the drug discovery process, thereby saving enormous late-stage development costs and allowing resources to be focused on the drugs most likely to succeed. Most critical in this regard is improving the suitability and robustness of the agents that enter the clinic.

Cancer Research UK Centre for Cancer Therapeutics, The Institute of Cancer Research, Haddow Laboratories, 15 Cotswold Road, Sutton, Surrey SM2 5NG, UK. Correspondence should be addressed to I.C. (ian.collins@icr.ac.uk) or P.W. (paul.workman@icr.ac.uk).

Published online 15 November 2006; doi:10.1038/nchembio840
Introduction of new technologies has been extremely importantparticularly various high-throughput genomic approaches for target and biomarker discovery ${ }^{2}$, high-throughput screening (HTS) for hit identification ${ }^{3,4}$ and structure-based design ${ }^{5}$, which we will discuss in detail. Although many individual developments are enhancing speed and quality, it is important to emphasize that most of the clinical successes so far have resulted from close integration of different technologies and disciplines (chemistry, biology and experimental medicine), and from the application of 'joined-up thinking', in particular to address the issues that lead to failure of drugs in the clinic ${ }^{1}$.

Chemical biology offers experimental techniques for linking together elements from all stages of what was previously viewed as a linear progression from gene to drug (Fig. 1). These techniques allow us to look ahead on the path to the clinic using probe compounds to refine the target pharmacological profile and select the best models to guide drug development $^{6}$. They also allow us to define the appropriate subject population for clinical trial by identifying biomarkers for drug action ${ }^{7}$. Chemical biology facilitates evaluation of compounds on a genome- or proteome-wide scale through interaction screens that examine many biological systems simultaneously in a well-defined manner, as in the elucidation of kinase inhibitor selectivity profiles ${ }^{8,9}$ and the identification of desirable polypharmacies and combination therapies through detection of synthetic lethality ${ }^{10,11}$.

Against this background, our review will focus on the discovery of new small-molecule anticancer drugs for relevant protein targets, highlighting particularly but not exclusively those acting on kinases and the molecular chaperone heat shock protein 90 (HSP90). These are areas of drug discovery in which chemical biology techniques are clearly making a significant impact. We assess the progress and current challenges of cancer drug discovery, and finally we indicate ways forward to enhance the discovery and development of cancer therapeutics. 


\section{The molecular basis of cancer: drugging the cancer genome}

Efforts to elucidate the molecular basis of cancer are not new. They date back to the characterization of animal cancer viruses in the 1960s and 1970s, the identification of the first cancer-causing oncogenes and tumor suppressor genes in the 1970s and 1980s and the discovery of the ways cancer genes subvert signal transduction pathways in the $1990 \mathrm{~s}^{12}$. What has been radically different in the last five to ten years is a profound cultural change, in which cancer drug discovery has embraced molecular oncology as a source of disease-causing targets for hypothesis-driven, mechanism-based drug discovery ${ }^{13,14}$.

The first generation of effective cancer drugs were the cytotoxics that still form the basis of most treatment regimens ${ }^{14}$. Many were discovered by screening for compounds that kill tumor cells. The concept underlying the development of these agents was that cancer cells replicate their DNA and divide more frequently than healthy cells. This somewhat naive notion underpinned the development of DNA-damaging agents, antimetabolites that inhibit DNA synthesis, and microtubule inhibitors such as Taxol (paclitaxel) that block the mechanics of cell division. Though this first era of cancer drug development did not deliberately exploit the genetic basis of cancer, many of the agents were nevertheless 'molecularly targeted'. For example, antifolate thymidylate synthase inhibitors were rationally designed according to principles of modern medicinal chemistry, which involved structure-activity relationships (SARs) and structural biology ${ }^{15}$. The term "targeted molecular therapeutics" and similar tags are now used to describe small-molecule agents that are not only rationally designed but also act on disease-causing oncogenic targets. The increasing number of approved agents, together with the therapeutic antibodies that act on similar targets (Table 1 and Box 1), demonstrates that we are in a second golden era of cancer drug development ${ }^{14}$.

The development of molecular cancer therapeutics is founded on an understanding of the types of genes involved (Fig. 2a). The process of exploiting cancer genes to develop both molecular therapeutics and molecular biomarkers is now well established (Fig. 2b). The integration of these forms the basis for the development of personalized cancer medicine $e^{13,14}$. Activation of oncogenes and inactivation of tumor suppressor genes—often facilitated by inactivation of DNA repair genes, which causes genetic instability-leads to hijacking of signal transduction pathways and hence to the various well-defined phenotypic hallmark traits of cancer ${ }^{16,17}$. These traits include not only loss of cell cycle control and the unrestricted proliferation referred to above, but also independence from positive and negative homeostatic regulatory

\section{BOX 1 THE NEED FOR IMPROVED CANCER THERAPIES AND IMPROVED DRUG DISCOVERY}

Despite many therapeutic successes, cancer is the second-mostfrequent cause of death in the United States and is set to become the most common in the relatively near future ${ }^{12}$. Cancer comes in many different forms - both anatomically and molecularly - and whereas in several of these (for example, some leukemias, lymphomas, testicular and pediatric cancer) drug therapy can markedly increase survival, in many of the common adult epithelial tumors the impact is modest at best.

The overall success with oncology drug development in recent years has been mixed, even though over 30 new cancer treatments have been approved by the US FDA since 2001 (http://www.centerwatch. com/patient/drugs/druglist.html; Table 1). Many of these approved drugs are antibodies, and others are not novel, first-in-class agents. Furthermore, attrition rates for oncology drugs in the clinic are worse than for other disease areas: figures for $1990-2000$ show a $5 \%$ success rate in the clinic, compared with $11 \%$ overall ${ }^{1}$. Moreover, failure often occurs very late in the clinical development process.

Reasons for the failure of candidate drugs for cancer and other diseases have been identified ${ }^{1}$. In 1991, poor pharmacokinetics and bioavailability predominated. Technical solutions (involving predictive assays to triage compounds with permeability and metabolic liabilities ${ }^{131}$ ) were implemented to address this, and by 2000 failure from this cause had fallen from $40 \%$ to $10 \%$. The main causes of attrition are now insufficient therapeutic activity (30\%) and toxicity (30\%). These risks can be reduced by identifying better predictive and molecularly defined animal models of cancer $^{99,100}$ and in vitro models of mechanism-based and offtarget toxicity ${ }^{101}$. However, drugs acting on new molecular targets are inherently risky. Risk can be minimized by selecting only the best targets ${ }^{22}$, and by using biomarkers to identify the most appropriate subjects ${ }^{110}$ and to demonstrate proof of concept for the intended mechanism of action? ${ }^{7}$.

Table 1 Examples of targeted molecular cancer therapeutics receiving marketing approval by the US FDA 2001-2006

\begin{tabular}{|c|c|c|c|c|}
\hline Year & Examples of targeted molecular therapeutics & Drug type & Disease indication & Primary molecular target \\
\hline \multirow[t]{4}{*}{2006} & Sprycel (dasatinib) & Small molecule & Gleevec-resistant CML & BCR-ABL, SRC \\
\hline & Sutent (sunitinib) & Small molecule & Renal cancer and GIST & PDGFR, VEGFR, C-KIT \\
\hline & Herceptin (trastuzumab) & Antibody & Breast cancer ${ }^{\mathrm{a}}$ & ERBB2 \\
\hline & Zolinza (vorinostat) & Small molecule & Percutaneous T-cell lymphoma & HDAC \\
\hline 2005 & Nexavar (sorafenib) & Small molecule & Renal cell carcinoma & VEGFR, CRAF, PDGFR \\
\hline \multirow[t]{3}{*}{2004} & Avastin (bevacizumab) & Antibody & Metastatic colorectal carcinoma & VEGF \\
\hline & Erbitux (cetuximab) & Antibody & EGFR-expressing metastatic colorectal cancer & EGFR \\
\hline & Tarceva (erlotinib) & Small molecule & Metastatic non-small-cell lung cancer & EGFR \\
\hline \multirow[t]{2}{*}{2003} & Iressa (gefitinib) & Small molecule & Metastatic non-small-cell lung cancer ${ }^{b}$ & EGFR \\
\hline & Velcade (bortezomib) & Small molecule & Multiple myelomac & $26 \mathrm{~S}$ proteasome \\
\hline \multirow[t]{2}{*}{2002} & Gleevec (imatinib) & Small molecule & GIST & C-KIT, PDGFR \\
\hline & Zevalin (90Y-ibritumomab tiuxetan) & Radiolabeled antibody & Non-Hodgkin lymphoma & CD20 \\
\hline \multirow[t]{2}{*}{2001} & Campath (alemtuzumab) & Antibody & B-cell chronic lymphocytic leukemia & CD52 \\
\hline & Gleevec (imatinib) & Small molecule & $\mathrm{CML}$ & BCR-ABL \\
\hline
\end{tabular}

aFirst approved 1998, use extended 2006. bSecond-line therapy. ${ }^{\circ}$ For people who have received at least two prior therapies. Also see CenterWatch (http://www.centerwatch.com/patient/drugs/ druglist.html). 
factors, inappropriate survival, decreased apoptosis, immortalization, and stimulation of invasion, angiogenesis and metastasis. All these processes present targets for therapeutic intervention. Oncogene products themselves may be good targets, but other proteins downstream in a key pathway may also be suitable; for example, MAP-kinase kinase 1 (MEK1) and MEK2 in the RAS-RAF-MEK-ERK signaling pathway and mammalian target of rapamycin (mTOR) in the phosphatidylinositol-3-OH kinase $(\mathrm{PI}(3) \mathrm{K})$ pathway. In addition, the use of chemical probes has shown that oncogenic support processes such as protein chaperoning (for example, HSP90) and chromatin regulation (for example, histone deacetylase (HDAC)) can provide valuable drug targets ${ }^{18,19}$.

A major expectation for targeted molecular cancer therapeutics is that they show good efficacy and low toxicity, and this is certainly true of the poster child Gleevec ${ }^{20}$. An important theory underpinning this selectivity is that of "oncogene dependence" or "addiction" 21. Although it requires further experimental validation, this concept proposes that cancer cells undergo selection to become driven by, but also dependent on, key oncogenic pathways.

A key driver in selecting cancer drug targets is the identification of distinguishing features of cancer cells, which may arise by mutations or gene rearrangements, inherited epigenetic changes or cell lineage legacies. While contributing to cancer development, these genetic, epigenetic or metabolic features can create "dependencies"-weaknesses that can be exploited according to four types or "tracks," as classified by Benson et al. ${ }^{22}$ : (i) the genetics track, based on oncogene addiction and exemplified by Gleevec in $B C R-A B L$-positive leukemia and MEK1 and MEK2 inhibitors in $B R A F$-driven melanoma models ${ }^{23}$; (ii) the synergy track, founded on the concept of synthetic lethality and illustrated by the selective killing by poly(ADP-ribose) polymerase (PARP) inhibitors of cells with $B R C A$ gene defects ${ }^{10}$; (iii) the lineage track, another form of addiction, based on gene expression profiles showing that cancers from a given tissue or cell of origin share many common molecular features of that origin, and exemplified by antihormonal therapies in breast and prostate cancer, and potentially by the amplified oncogene microphthalmia-associated transcription factor $(M I T F)$ in aggressive melanomas ${ }^{24}$; and (iv) the host track, arising from recognition that tumor-host cell interactions and microenvironmental and physiological factors related to tumor hypoxia are critical for cancers, and exemplified by the vascular endothelial growth factor (VEGF)-targeted antibody Avastin and smallmolecule inhibitors of VEGF receptor tyrosine kinases such as Nexavar (sorafenib) and Sutent (sunitinib). Together with the view of molecular cancer therapeutics discussed above (Fig. 2), the concept of cancer dependencies provides a valuable framework for thinking about oncology drug targets, selecting an individual target to work on, or building a portfolio of targets to tackle the disease in different ways.

There is certainly no shortage of potential drug targets. More than 350 cancer genes have been catalogued (www.sanger.ac.uk/genetics/ CGP/Census/ $)^{25}$. New cancer genes continue to be found, particularly by high-throughput systematic methods such as genome resequencing ${ }^{26}$ (as used to discover $B R A F^{27}$ ) and array-based DNA copy number and expression-profiling analysis (as used to discover $M I T F^{24}$ ). A short hairpin RNA ( shRNA) barcode screen has been used to identify genes that affect sensitivity to the nutlin inhibitors of p53-binding MDM2 (ref. 28), and a small interfering RNA (siRNA)-based screen has identified kinases that cooperate with AKT (protein kinase B) ${ }^{11}$. High-throughput RNA interference (RNAi) technology is a powerful tool for gene discovery ${ }^{29}$. Validation and prioritization of the best targets is now a critical activity. This can be done using a combination of human genetics and genomics; functional validation, especially by overexpression or knockdown by RNAi; and transgenic animals and model organisms ${ }^{22}$. Extremely important in triaging targets is their druggability. For example, with current technology, enzymes such as kinases and small-domain-size protein-protein interactions are druggable, but many potential targets are not, as with the mutant oncoprotein RAS and mutant tumor suppressor p53.

Genes hijacked in cancer have important homeostatic roles in development and normal physiology. Despite cancer dependencies, toxicity to healthy cells is possible. For example, epidermal growth factor receptor (EGFR)-targeted agents cause mechanism-based skin rash. In retrospect, skin toxicity might have been predicted from the phenotype of EGFR knockout mice, was seen in the preclinical toxicology of Iressa (gefitinib) and Tarceva (http://www.accessdata.fda.gov/scripts/cder/drugsatfda/), and can now be understood in terms of the receptor tyrosine kinase ERRB family biology ${ }^{30}$. However, therapeutic index remains difficult to predict accurately purely from knowledge of the molecular action of 
a

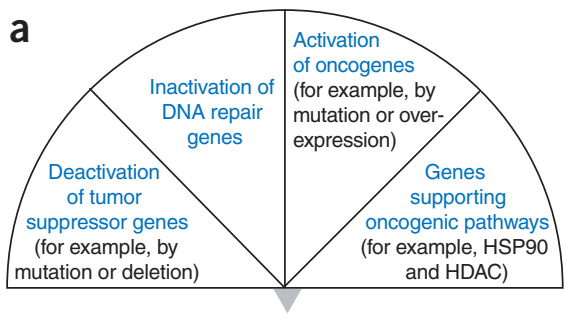

Stimulation of oncogenic signal transduction pathways

Phenotypic hallmarks of cance

- Increased proliferation

- Inappropriate survival/decreased apoptosis

- Immortalization

- Invasion, angiogenesis and metastasis b

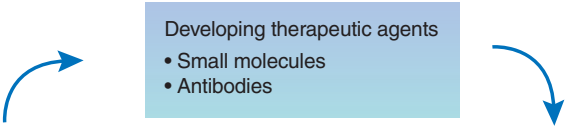

Elucidating the cancer genome

- Discovery of all genes involved in cancer

- Validation of these or related genes as drug targets
Personalized cancer medicine

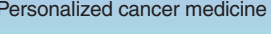

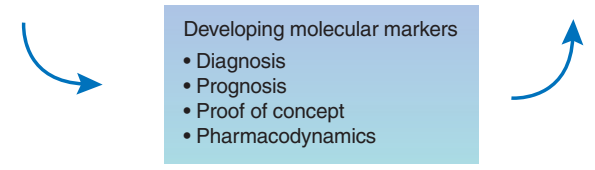

\section{Cancer}

Figure 2 Understanding the genetic and biological causation of cancer generates new targets for therapy. (a) Schematic illustration of the classes of genes involved in oncogenic transformation and malignant progression. Deregulation of these genes leads to the hijacking of oncogenic signaling pathways, which results in the phenotypic hallmark traits of cancer. Mutated or overexpressed oncogenes, particularly kinases, are often excellent targets for drugs that act as enzyme inhibitors. Downstream signal transduction proteins can also be inhibited (for example, MEK1 and MEK2 inhibitors acting downstream of RAS and RAF). Replacing the loss of tumor suppressor genes (for example, p53) is very challenging, and restoring a loss of function with a small molecule is also extremely difficult. However, loss of tumor suppressor activity can lead to a gain of function elsewhere (for example, activation of the PI(3)K pathway when the function of the tumor suppressor PTEN is lost), and this function can be inhibited. Loss of DNA repair genes can be exploited by using inhibitors of other

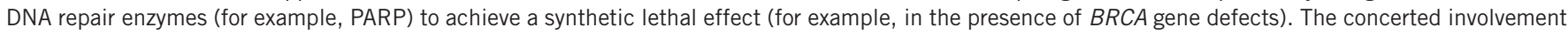
of several genes in the progression of most cancers suggests that a combinatorial approach may prove to be the most effective. This could be achieved with cocktails of highly selective drugs, with multitargeted or polypharmacy agents, or by inhibiting targets such as HSP9O and HDAC that in turn affect many cancer gene products. (b) Translating knowledge about the molecular basis of cancer into individualized cancer treatment, which requires both drugs and biomarkers. Modified from ref. 13 with permission.

drugs and is probably still best estimated from animal models of toxicity and efficacy during preclinical development.

\section{Lead generation: finding the diamond in the rough}

The next major step after target selection is lead generation (Fig. 1). Chemical starting points for drug discovery may come from the structures of endogenous ligands, existing drugs, biologically active natural products, high-throughput or focused screening of synthetic-compound libraries and, increasingly, design and screening using structural biology information. Biochemical HTS against isolated cancer targets has been effective for several protein classes, including kinases ${ }^{3}$ and HSP90 ${ }^{18}$. For kinases, such screens can be biased toward identifying competitive inhibitors of phosphate transfer from ATP by the active enzyme. In contrast, in vitro biochemical recapitulation of signal transduction cascades (for example, the RAF-MEK-ERK pathway $^{31}$ ) may identify compounds having a wider range of well-defined mechanisms of action.

Phenotypic screening in intact cells or organisms, such as Caenorhabditis elegans or zebrafish embryos, is applicable to target identification and lead generation ${ }^{4}$. This extends the reach of small-molecule screening to targets that are incompatible with in vitro biochemical assays and allows many molecular targets to be probed simultaneously in the cellular environment, as in the discovery of an inhibitor of oncogenic $\mathrm{WNT} / \beta$-catenin signaling ${ }^{32}$. Postscreening mechanistic deconvolution of active compounds is needed to define their precise molecular targets $^{33}$. For noncovalent inhibitors this may involve gene expression and protein array profiling, affinity chromatography of cell lysates, or yeast chemical genetic screens, as used to find the targets of antiproliferative phenylaminopyrimidines related to Gleevec ${ }^{34}$. Chemically reactive inhibitors can be used directly as labels to isolate their targets, as shown for inhibitors of breast cancer cell proliferation ${ }^{35}$. Alternatively, imaging-based high-content screens interrogate the phenotype directly at the molecular level. Imaging of protein recruitment to the cell membrane has identified inhibitors of AKT kinase activity ${ }^{36}$ and has determined the effects of selective $\mathrm{PI}(3) \mathrm{K}$ inhibitors on the response to growth factors $^{37}$. Observation of subcellular localization of the transcription factor FOXO1A has identified small molecules targeting the PI(3)K-AKT pathway and nuclear transport machinery ${ }^{38}$.

Sensitive biophysical techniques such as NMR and X-ray diffraction can detect the weak binding of much smaller compounds (fragments) than those comprising typical HTS libraries. Analysis of fragment binding modes may suggest how to link the fragments to generate more traditional lead structures ${ }^{39}$, as shown for p38 MAP-kinase inhibitors ${ }^{40}$. Combining biochemical HTS for low-affinity compounds and mediumthroughput biophysical methods, especially protein-ligand cocrystallography, is a powerful and efficient lead-generation strategy ${ }^{41}$.

Computational chemistry increasingly contributes to screening strategies. Empirical parameters describing appropriate physicochemical properties of fragments, leads and drugs should be routinely incorporated into new compound library design ${ }^{42-47}$ (Box 2). Some structural motifs confer promiscuous nonspecific activity, or are associated with toxicities, and hence can also be eliminated from screening sets ${ }^{48,49}$. Virtual screening ${ }^{50}$ of library structures for their fit with specific threedimensional pharmacophores can enrich HTS hit rates, as shown for inhibitors of checkpoint kinase 1 (ref. 51). Pharmacophores may come from protein-inhibitor cocrystal structures, or they may be derived from SARs.

The most efficient screening strategy depends on the molecular target and what is known when the drug discovery program starts. The various approaches may require compound libraries of quite different designs. At one extreme, large collections of highly diverse structures are useful for biochemical HTS against targets for which there is little chemical biology information. Libraries biased with compounds having a relevant biological pedigree increase the hit rates in phenotypic screens, as with RAS signaling pathway inhibitors ${ }^{52}$. When structural biology or 
known ligands define a pharmacophore, smaller, focused libraries based on a few structural motifs may be useful. The close structural similarity of the ATP cofactor binding site across the kinome enables the development of privileged structures or molecular master keys (molecular scaffolds with a good fit and therefore high probability of binding), which provide starting points for new inhibitor discovery and allow target hopping within a chemical class ${ }^{53,54}$. One potential weakness is the increased probability of finding kinase cross-reactive 'frequent hitters' and the consequent need to engineer selectivity into compounds during drug development.

The clinical anticancer activity of complex natural products-for example, camptothecins, vinca alkaloids and epothilones-suggests that such structures occupy a pharmaceutically valid chemical space $e^{55}$. Natural-product core scaffolds may be a source of diverse new chemical materials that serve as starting points for developing targeted therapeutics. Biology-oriented ${ }^{56}$ and diversity-oriented synthesis ${ }^{57}$ are emerging paradigms for guiding the generation of compound libraries that mimic the structural complexity of natural products, outside the arguably restricted chemical space occupied by synthetic drugs ${ }^{58}$. However, the extent to which highly complex structures are compatible with druglike physicochemical properties is not fully determined ${ }^{59}$. It will also be important to distinguish molecularly targeted biological activities from general cytotoxic activity when natural products serve as inspiration for anticancer drug discovery.

\section{Structure-based approaches: designer drugs}

As mentioned before, the engine of medicinal chemistry continues to be the iterative cycles of design, chemical synthesis and biological evaluation that establish SARs. Observation of the interaction of ligands and proteins through cocrystallography informs and accelerates the process and has been very successful in kinase, HSP90 and HDAC inhibitor design. Crystallographic identification of potential small-molecule binding sites embedded in the larger surfaces that usually mediate proteinprotein interactions has been important in discovering proapoptotic agents that inhibit interactions of MDM2-p53 and BCL2 ${ }^{60}$.

Structural biology has revealed several binding modes for protein kinase inhibitors ${ }^{5,61}$ (Fig. 3a-d). Behind these is the conserved bilobal tertiary structure defining a binding cleft for the cofactor ATP. Although the sequence and structural similarity of kinases was originally viewed as a potential liability, they are now recognized as classic druggable targets $^{62}$. The binding site contains conserved features that enable hydrogen bonding to the adenosine, ribose and phosphate components of ATP. These areas are flanked by subsites that are not occupied by cofactor and that vary considerably between kinases. Inhibitors exploit conserved and nonconserved features to achieve potency and selectivity. Additionally, conformational changes (usually triggered by phosphorylation) associated with activation of kinases indicate that each kinase target may exist in more than one form. Drugs such as Iressa and Tarceva compete for occupation of the ATP binding site in the activated enzyme, whereas Gleevec, Nexavar and Tykerb (lapatinib) bind and stabilize an inactive form, thereby preventing activation ${ }^{5,61}$. Other compounds, such as the MEK1 and MEK2 inhibitor PD318088 (ref. 63), bind remotely and allosterically inhibit enzyme activity. Inhibition may also involve binding to sites that mediate protein-protein interactions, as seen for ligands of the AKT pleckstrin-homology domain ${ }^{64}$ and the mTOR inhibitor rapamycin ${ }^{65}$.

The structural biology of kinases has important consequences for inhibitor selectivity and development of resistance. Three-dimensional structure is more obviously conserved between activated kinases than between inactive forms. Thus inhibitors targeting stabilization of inactive enzymes can be more selective $\mathrm{e}^{5,61}$. Greater use of interactions within the nonconserved regions of the ATP binding site should confer greater selectivity for inhibitors targeting active forms. However, the potential for drug resistance should also be considered. Mutation of residues essential for binding of drugs to the inactive form may be better tolerated in terms of retaining catalytic function in the active conformation. Likewise, regions of the ATP binding site that are not involved in catalysis or cofactor binding are likely to be hot spots in which mutations do not affect kinase function but may ablate drug binding. The gatekeeper residue, which delineates the size of a buried pocket adjacent to the ATP binding site and is mutationally silent in terms of ATP binding, is one such residue. Gatekeeper and other mutational hot spots have been identified in variants of Gleevec-resistant BCR-ABL ${ }^{66}$ and Iressa-resistant EGFR kinase ${ }^{67}$. Deliberate mutation of kinase residues is a valuable tool for target validation and investigation of drug action. Construction of a gatekeeper mutant of EGFR that is insensitive to Iressa paralled the identification of the EGFR T790M Iressa-resistant mutant in clinical trials ${ }^{68}$. The effect of conditional kinase inhibition on signaling pathways

\begin{abstract}
Figure 3 Protein-ligand cocrystal structures showing the canonical protein folds and inhibitor binding modes for protein kinases, HSP9O and HDAC. Ribbon representations of the proteins are colored with respect to their secondary structure: red, $\alpha$-helix; blue, $\beta$-sheet; green, turn structures. Space-filling representations of the inhibitors are colored by electrostatic potential: red, negative charge; blue, positive charge; gray, neutral. (a) ATP (space filling) bound to PKA (Protein Data Bank (PDB) entry 1ATP). (b) Tarceva (space filling) bound to EGFR in an active conformation (PDB entry 1M17). (c) Gleevec (space filling) bound to $B C R-A B L$ in an inactive conformation (PDB entry 1IEP). (d) PD318088 (space filling) bound to MEK1 (PDB entry 1S9J); ADP (orange ball and stick) is also bound. (e) Geldanamycin (space filling) bound to yeast Hsp90 (PDB entry 1A4H). (f) CCT018159 (space filling) bound to yeast Hsp90 (PDB entry 2BRC). (g) SAHA (space filling) bound to HDAC8 (PDB entry 1T69). The active site forms a deep tubular pocket that is filled by the inhibitor.
\end{abstract}

a $\quad b$

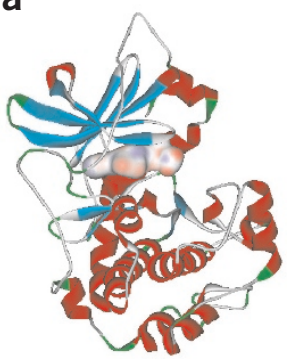

e

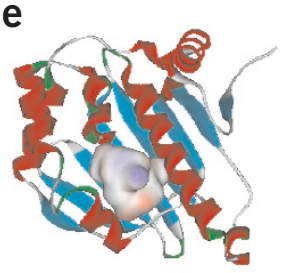

b

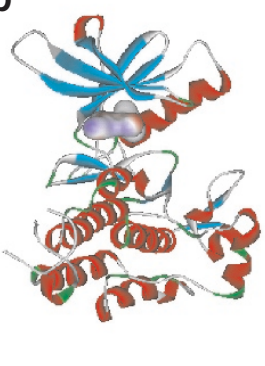

C

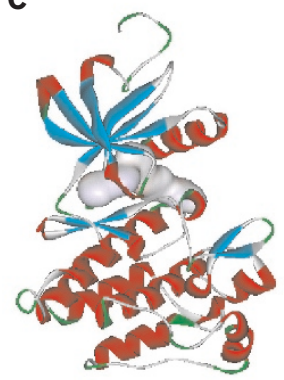

f

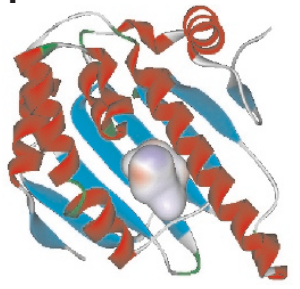

d

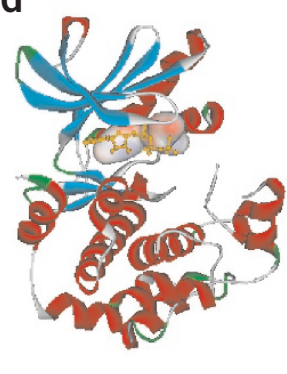

g

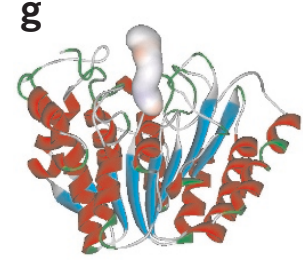




\section{BOX 2 DIFFERENCES IN THE PROPERTIES OF FRAGMENTS, LEADS AND DRUGS}

Not all chemical structures are equal in terms of their likely progression to a drug candidate. The chemical outputs of screening programs (hits) are evaluated for synthetic and physicochemical tractability and for the presence of useful SARs through the synthesis of a limited number of analogs (hit to lead). In addition to target potency and selectivity, cellular efficacy and preliminary ADMET properties are evaluated for promising compounds (lead profiling) to select the best chemical starting point (lead) ${ }^{80}$. Lead optimization typically involves addition of chemical functionality to improve the potency, selectivity and in vivo activity of compounds ${ }^{44}$. To accommodate this increase in molecular weight, good-quality leads are generally smaller and less lipophilic and contain less functionality than typical drugs. This ensures that the larger, optimized compound will still be drug-like (defined as the balance of physicochemical properties needed to achieve adequate bioavailability through aqueous solubility and transit through lipid membranes) ${ }^{42}$. Fragments are smaller and simpler than traditional leads, as they occupy only a fraction of the druggable binding site on the target ${ }^{39}$. Table 2 summarizes the typical properties of fragments, lead-like and drug-like molecules suggested in the medicinal chemistry literature.

Table 2 The properties defining fragments, leads and drugs

\begin{tabular}{|c|c|c|c|}
\hline & Fragment 39 & Lead $^{43,44,80}$ & Drug $42,43,45-47$ \\
\hline \multicolumn{4}{|l|}{ Physicochemical } \\
\hline Molecular weight (Da) & $<300$ & $<400-450$ & $<500$ \\
\hline $\mathrm{H}$-bond donors $(\mathrm{OH}, \mathrm{NH})$ & $\leq 3$ & $\leq 4-5$ & $\leq 5$ \\
\hline $\mathrm{H}$-bond acceptors $(\mathrm{N}, \mathrm{O})$ & $\leq 3$ & $\leq 8-9$ & $\leq 10$ \\
\hline Polar surface area $45-47$ & NA & NA & $\leq 140-150 \AA^{2}$ \\
\hline Chemically reactive groups 49 & NA & None present & None present \\
\hline \multicolumn{4}{|l|}{ Biological } \\
\hline Target activity $\left(\mathrm{IC}_{50} \text { or } K_{\mathrm{i}}\right)^{84}$ & $>10^{-5} \mathrm{M}$ & $10^{-6}-10^{-7} \mathrm{M}$ & $10^{-8}-10^{-9} \mathrm{M}$ \\
\hline Ligand efficiency ${ }^{85}\left(\mathrm{kcal} \mathrm{atom}^{-1}\right)$ & $>0.3$ & $>0.3$ & $>0.3$ \\
\hline SAR & NMR or X-ray data & Useful SAR established & Full SAR understood \\
\hline Toxicology & No common toxophores present & $\begin{array}{l}\text { No problem off-target pharmacology, } \\
\text { for example HERG }\end{array}$ & Therapeutic window \\
\hline
\end{tabular}

NA, not analyzed / not applicable; IC 50 , half-maximal inhibitory concentration; CYP450, cytochrome P450; i.v., intravenous; p.o., oral.

can be probed by kinase mutants that are sensitive to chemically orthogonal inhibitors not affecting the wild type ${ }^{69}$.

Some kinases contain a cysteine residue in the active site. Starting from the chemical scaffolds of reversible inhibitors such as Iressa, structurebased design has enabled development of irreversible inhibitors, such as the anilinoquinazoline CI-1033, that have low nonspecific chemical reactivity and that covalently attach to the thiol group ${ }^{70}$. A chemical genetics approach has led to highly selective, irreversible inhibitors through targeting the limited number of kinases in which the gatekeeper residue and a reactive cysteine are close in the ATP site ${ }^{71}$.

Structure-based design has been valuable in identifying and optimizing HSP90 inhibitors. The structure of the natural products geldanamycin and radicicol bound to the $\mathrm{N}$-terminal ATPase domain of HSP90 revealed the presence of a unique folding pattern in the ATP binding site that includes a network of tightly bound water molecules ${ }^{72}$ (Fig. 3e). Cocrystallization of the arylpyrazole HTS hit CCT018159 has shown that the resorcinol motif of this small molecule exploits the water network in a manner similar to that of radicicol $^{73}$ (Fig. 3f). The first synthetic inhibitors of HSP90 ATPase, a series of purines, were developed from modeling based on HSP90-ATP ${ }^{74}$, though subsequent cocrystallography of the inhibitor PU3 revealed an unexpected change in conformation that creates a new binding pocket ${ }^{75}$. Iterative reinves- tigation of ligand-protein structures during lead optimization is highly valuable for understanding the conformational variability of the target and its consequence for drug design.

Structure determination of a bacterial homolog of HDAC has defined the interaction of the inhibitor suberoylanilide hydroxamic acid (SAHA); it shows the hydroxamate group chelating an enzyme-bound zinc atom at the base of a deep tubular pocket ${ }^{76}$ (Fig. 3g). Elucidation of human HDAC8 structures has shown that the surface adjacent to the conserved active site pocket is conformationally malleable ${ }^{77}$, thereby presenting opportunities and challenges for design of HDAC subtypeselective inhibitors ${ }^{78}$. Chemical genetic screening has unraveled the mechanism and possible polypharmacy of HDAC inhibitors, with different isoforms catalyzing acetylation of histones versus tubulin ${ }^{79}$.

\section{Multiparameter lead optimization: polishing the diamond}

With the widespread use of HTS in many therapeutic areas from the late1980s onwards came the realization that not all small-molecule leads are suitable for optimization to drug candidates (Box 2). Profiling of potential leads against multiple chemical, physicochemical and biological criteria was adopted to select the best chemical starting point and biological test cascade to maximize the probability of clinical success $^{80}$. Multiparameter profiling continues iteratively throughout lead 
optimization to identify compounds having an acceptable profile across the whole range of properties needed for an effective drug.

In addition to considerations of physicochemical properties, synthetic tractability of leads is also important, as many structurally diverse analogs are needed during optimization. Fortunately, combinatorial and parallel syntheses used for contemporary screening libraries are usually adaptable for lead optimization ${ }^{81}$, as exemplified in the development of Nexavar ${ }^{82}$. Advances in synthetic and medicinal chemistry contribute to the discovery of new biologically active scaffolds, as in the incorporation of boronic acid functionality into the US Food and Drug Administration (FDA)-approved proteasome inhibitor Velcade (bortezomib) ${ }^{83}$.

Potency against the molecular target is an obvious consideration, with respect to both biochemical and cellular readouts ${ }^{84}$. It is useful to compare leads by ligand efficiency, a measure of the effectiveness of the interaction of the chemical structure with the target ${ }^{85}$. A key goal is to establish productive SARs, in which changes to the lead structure elicit corresponding improvements in biological activity.

Determining selectivity for the molecular target is very important and requires a combination of biochemical and cellular approaches. One issue is the extent to which comparison of in vitro measurements of compound activity at various isolated targets accurately reflects selectivity in cells, even with proteins from the same family, such as kinases $^{86}$. Inherent differences in kinase expression levels, enzyme kinetics and responsiveness of downstream effectors along individual pathways may lead to selective phenotypic outcomes from apparently unselective inhibitors. For example, despite a 20 -fold in vitro selectivity for inhibition of cyclin-dependent kinase 1 (Cdk1), GW297361 elicits a response in yeast cells indicative of selective inhibition of pho85 over Cdk $1^{87}$. Nevertheless, in vitro selectivity profiling using panels of isolated kinases ${ }^{88}$, or other enzymes and receptors, remains a valuable tool for comparing overall specificity. Other methods of kinase selectivity profiling parallel proteomic and chemical genetic techniques for identifying targets of phenotypic screens.

Figure 4 Kinase binding selectivity for representative inhibitors shown on the human kinome dendrogram. Selectivity was determined by competitive binding to 113 kinase enzymes expressed in bacteriophage ${ }^{9}$. Inhibitors may range from highly selective, such as Tykerb and Gleevec, through a targeted polypharmacology as shown for Nexavar, to the pan-kinase inhibition shown by staurosporine. Importantly, the structural homology of the kinase domain across the kinome means that inhibitors may show cross-reactivity not only for closely related enzymes, but also for unrelated kinases. Designing polypharmacology of kinase inhibitors in a controlled manner is a significant research challenge in this area. Modified from ref. 9 with permission. The kinase dendrogram was adapted and reproduced with permission from Science and Cell Signaling Technology, Inc. (http://www.cellsignal.com). With sponsorship by Cell Signaling Technology and Sugen, the figure was originally presented as a poster in Science to accompany the first analysis of the complete human kinome. TK, nonreceptor tyrosine kinases; RTK, receptor tyrosine kinases; TKL, tyrosine kinase-like kinases; CK, casein kinase family; PKA, protein kinase A family; CAMK, calcium/calmodulin-dependent kinases; CDK, cyclin-dependent kinases; MAPK, mitogenactivated protein kinases; CLK, CDK-like kinases.
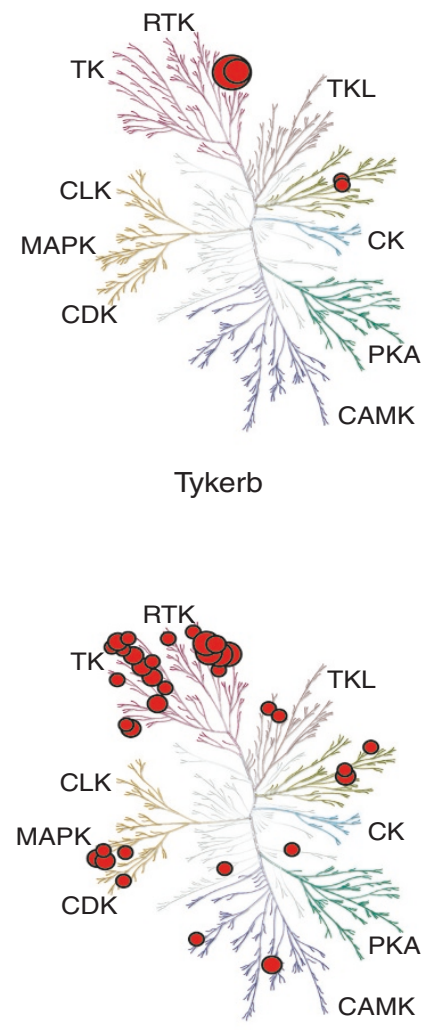

Nexavar
Affinity chromatography has revealed the cellular targets of Iressa ${ }^{89}$ and hymenialdisine $\mathrm{e}^{90}$, and a yeast chemical genetic screen has identified the targets of CDK inhibitors ${ }^{8}$. Maps of the interactions of clinical kinase inhibitors with 113 kinases have been assembled based on competitive binding of free inhibitors and immobilized, unselective probe inhibitors to bacteriophage-expressed kinases ${ }^{9}$ (Fig. 4).

As mentioned earlier, adopting strategies to evaluate pharmacokinetic properties as early as possible in drug discovery has substantially reduced clinical failure due to inadequate bioavailability ${ }^{1}$. In contrast to cytotoxic compounds, molecular cancer therapeutics may generally require chronic, and therefore oral, dosing. Lead profiling and optimization therefore need to concentrate on the absorption, distribution, metabolism, excretion and toxicity (ADMET) of compounds. This is facilitated by medium- and high-throughput in vitro models, although these require validation for each structural class to ensure that they accurately reflect in vivo behavior. Compound bioavailability depends on two contradictory physicochemical requirements - aqueous solubility and lipid membrane permeability — which must be balanced in an effective pharmaceutical. High-throughput assays for passive diffusion across artificial lipid membranes and turbidity assays for solubility are valuable ${ }^{91}$. Measurement of transport across Caco- 2 cell monolayers ${ }^{92}$ informs on permeability, active transport and, importantly, susceptibility to drug efflux pumps, which can compromise bioavailability and are a key factor in tumor resistance to chemotherapies ${ }^{93}$. Compound metabolism in microsomal preparations or hepatocytes identifies structures likely to be rapidly metabolized in vivo, whereas metabolite identification by mass spectrometry may suggest modifications to block this ${ }^{94}$. Avoiding the potential for compounds to interfere with therapeutic concentrations of other drugs by inhibition or induction of the drug-metabolizing cytochrome $\mathrm{P} 450$ enzymes is also important ${ }^{95}$. In vivo cassette dosing, 
in which small sets of compounds are dosed simultaneously, reduces animal usage and provides faster feedback of pharmacokinetic information, as illustrated by recent experience with purine CDK2 inhibitors and arylpyrazole HSP90 inhibitors ${ }^{96,97}$.

The goal of lead optimization is a drug candidate for clinical evaluation. Demonstrating efficacy and a therapeutic window in relevant in vivo models is therefore very important. Compounds with potent biochemical and cellular activity, and adequate pharmacokinetic properties, are progressed to in vivo models. Initial studies should focus on recapitulating a cellular pharmacodynamic response in the intact tumor and correlating this with tumor concentrations of compounds to establish pharmacokinetic-pharmacodynamic relationships ${ }^{98}$. In some cases, poor pharmacokinetics and low exposure to compound may be compensated for by long duration of action (slow off-rate) at the specific target ${ }^{84}$, thereby leading to a viable pharmacodynamic response. However, inherent poor pharmacokinetic properties leave no room for maneuver during clinical development and therefore represent a considerable risk to the eventual success of the compound ${ }^{1}$. Prioritized compounds that pass through a pharmacokinetic-pharmacodynamic filter are generally evaluated for efficacy in tumor growth inhibition in molecularly characterized models (for example, human tumor xenografts in rodents) ${ }^{99}$. Transgenic models are also valuable for proof of concept but are generally less convenient and reproducible for lead optimization ${ }^{100}$. In addition to tolerability and toxicity in animals, many specific off-target activities causing toxicological issues in humans can now be investigated by in vitro screening ${ }^{101}$. Some of these are general problems, such as inhibition of the HERG cardiac ion channel, whereas others may be associated with particular chemical scaffolds, for example the rich pharmacology of purine-based ligands ${ }^{102}$.

\section{Selected case histories: chemical biology in action}

Development of molecular-targeted agents for kinases, HSP90 and HDACs illustrates both lead optimization and the importance of feedback from clinical studies to the early drug discovery process. Formation of the BCR-ABL fusion protein is a specific oncogenic event for chronic myeloid leukemia (CML). Highly selective BCR-ABL inhibitors were generated from a series of anilinopyrimidine protein kinase C (PKC) inhibitors, an example of target hopping. Lead optimization to yield Gleevec focused on improving pharmacokinetic properties ${ }^{20}$ (Scheme 1a). The success of Gleevec in clinical trials for CML provided an important proof of concept for the development of molecular-targeted smallmolecule drugs in oncology. The observation that Gleevec inhibits a limited number of other kinases, notably the oncoprotein c-KIT, has led to successful clinical trials of the drug for the treatment of gastrointestinal stromal tumor (GIST), which is often driven by a mutant kinase ${ }^{103}$. The emergence of Gleevec-resistant kinase mutants in people with CML has prompted the development of second-generation inhibitors such as Sprycel (dasatinib), which targets the more conserved active form of the kinase and inhibits many of the Gleevec-resistant mutants as well as other targets such as the nonreceptor tyrosine kinase SRC ${ }^{104}$.

a
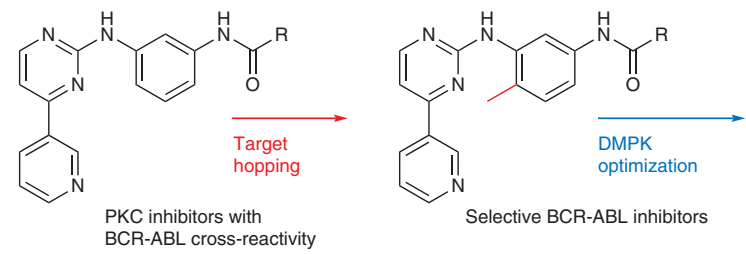

Selective BCR-ABL inhibitors

b<smiles>COC(=O)c1sc(C(C)(C)C)cc1NC(=O)Nc1ccccc1</smiles>

CRAF inhibitor from HTS $\left(\mathrm{IC}_{50}=17 \mu \mathrm{M}\right)$

C<smiles>CCc1cc(-c2n[nH]c(C)c2-c2cccc3c2OCCO3)c(O)cc1O</smiles>

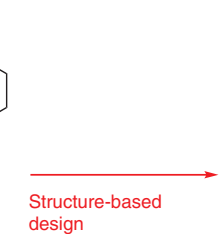

СCT018159

HSP90 inhibitor from HTS $\left(\mathrm{IC}_{50}=9 \mu \mathrm{M}\right)$

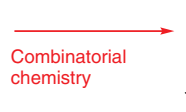
design S

S

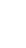
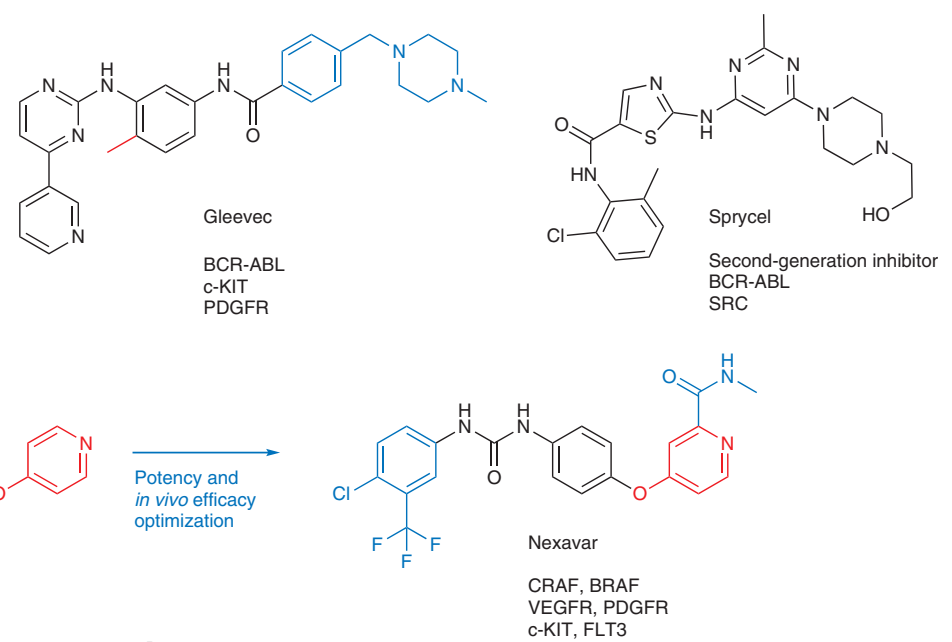<smiles>CCNC(=O)c1[nH]nc(-c2cc(Cl)c(O)cc2O)c1-c1ccc(OC)cc1</smiles>

VER49009
Potent, cell-active HSP90 inhibitor $\left(\mathrm{IC}_{50}=25 \mathrm{nM}\right)$ d
NVP-LAK974 HDAC inhibitor from HTS

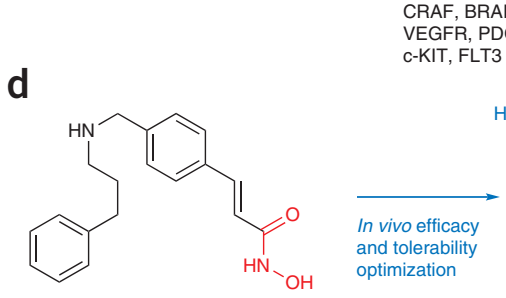

\section{Potency and} in vivo efficacy optimization and tolerability
optimization

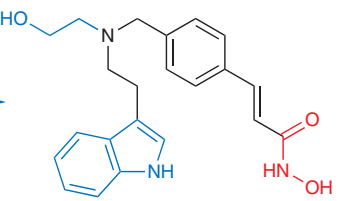

NVP-LAQ824 Clinical candidate HDAC inhibitor

Scheme 1 Selected case histories of lead generation and lead optimization for small-molecule-targeted molecular cancer therapeutics. (a) The phenylaminopyrimidine core of Gleevec emerged from screening of PKC inhibitors and was rendered selective for BCR-ABL by addition of a single methyl substituent (red). Lead optimization focused on improving pharmacokinetic properties (blue). The second-generation BCR-ABL inhibitor Sprycel has activity against Gleevec-resistant BCR-ABL mutant kinases. DMPK, drug metabolism and pharmacokinetics. (b) The starting point for the discovery of Nexavar came from HTS of a large compound collection against CRAF. Combinatorial variation of the two substituents on the central urea generated a potent lead (red). Lead optimization focused on improving potency and in vivo activity (blue). (c) HTS against the HSP90 ATPase identified the novel pyrazole inhibitor CCT018159, which was cocrystallized with the enzyme. Structure-based design guided the positioning of extra lipophilic and hydrogen-bonding functional groups to generate the potent, cell-active inhibitor VER49009 (red). (d) The HDAC inhibitor NVP-LAK974 was identified by HTS of a large compound collection. It contains the hydroxamate zinc-binding functionality typical of many HDAC inhibitors (red). Lead optimization to the clinical candidate NVPLAQ824 concentrated on improvements to in vivo activity and tolerability (blue). 
Nexavar exemplifies the use of combinatorial chemistry for lead generation and optimization from a privileged structure, the diarylurea ${ }^{82}$ (Scheme 1b). Although developed as a targeted CRAF inhibitor, Nexavar was subsequently recognized as having useful receptor tyrosine kinase polypharmacology (particularly in inhibiting VEGFR, platelet-derived growth factor receptor (PDGFR), c-KIT and FMS-related tyrosine kinase 3 (FLT3)), which led to its approval for the treatment of renal cell cancers ${ }^{105}$. Single-agent activity was not, however, seen in melanoma, despite the fact that Nexavar does have activity on BRAF. Selectively targeting oncogenic BRAF is one focus for research on second-generation RAF inhibitors ${ }^{31}$.

The search for small-molecule inhibitors of HSP90 was given impetus by successful proof-of-concept clinical trials with 17-AAG, a derivative of the natural product geldanamycin ${ }^{18}$, which showed both the molecular signature of target inhibition in tumor tissue and evidence of activity in individuals with melanoma, but which has solubility, formulation and metabolic liabilities ${ }^{106}$. The optimization of the 3-(2,4dihydroxyphenyl)pyrazole HTS hit CCT018159 (ref. 72) exemplifies the way in which protein structure information guides the choice and positioning of extra functionality to improve inhibitor affinity. The amidopyrazole VER49009 is one of the first small-molecule HSP90 inhibitors to be described that demonstrates the required potency to become a clinical candidate ${ }^{107}$ (Scheme 1c).

Another area in which early clinical trials of a natural-product inhibitor, Zolinza (vorinostat, SAHA), have informed subsequent small-molecule drug discovery is the development of HDAC inhibitors $^{108}$. Zolinza has been approved for use in percutaneous T-cell lymphoma but has suboptimal pharmacokinetics. The HTS hit NVPLAK974 contains a novel cinnamyl hydroxamate as the essential zincchelating group common to the inhibitor class (Scheme 1d). In this example, lead optimization concentrated on improving in vivo efficacy and tolerability, thereby leading to the clinical candidate NVPLAQ824 (ref. 109).

\section{Biomarkers: the pharmacologic audit trail}

Molecular diagnostics are required to identify individuals most likely to benefit from molecularly targeted therapy ${ }^{110}$. Molecular biomarkers are also needed for proof of concept of target inhibition and for optimizing dosing schedules ${ }^{7}$. Biomarkers are used to make clinical trials more intelligent and informative, and to make decision making more rational and effective 110,111 . We have developed the concept of the 'pharmacologic audit trail', which offers a logical and practical framework for tracking the performance of a drug during both preclinical and clinical development ${ }^{7,13,112,113}$. It also provides a rational basis for assessing the risk of failure and for making important decisions on project progression. The pharmacologic audit trail consists of a series of hierarchically or sequentially arranged questions (Fig. 5).

Ideally data should be collected at each level. If the answer is "no" at any point then immediate action should be taken, with one possibility being termination of the project. As a drug progresses through the hierarchy of questions the risk of failure decreases. It is essential to have a series of robust, validated assays available for molecular biomarkers and pharmacokinetic behavior. Advances in genomic technologies have enhanced biomarker discovery ${ }^{114}$. The development of minimally invasive methods based on positron emission tomography and NMR spectroscopy or imaging is particularly important ${ }^{115}$. An important point in biomarker-driven decision making is that quantitative information is frequently lacking concerning exactly how hard a target or pathway needs to be hit to obtain a relevant degree of therapeutic benefit. This needs to be better defined in preclinical models using quantitative technologies, such as ELISA ${ }^{116}$.

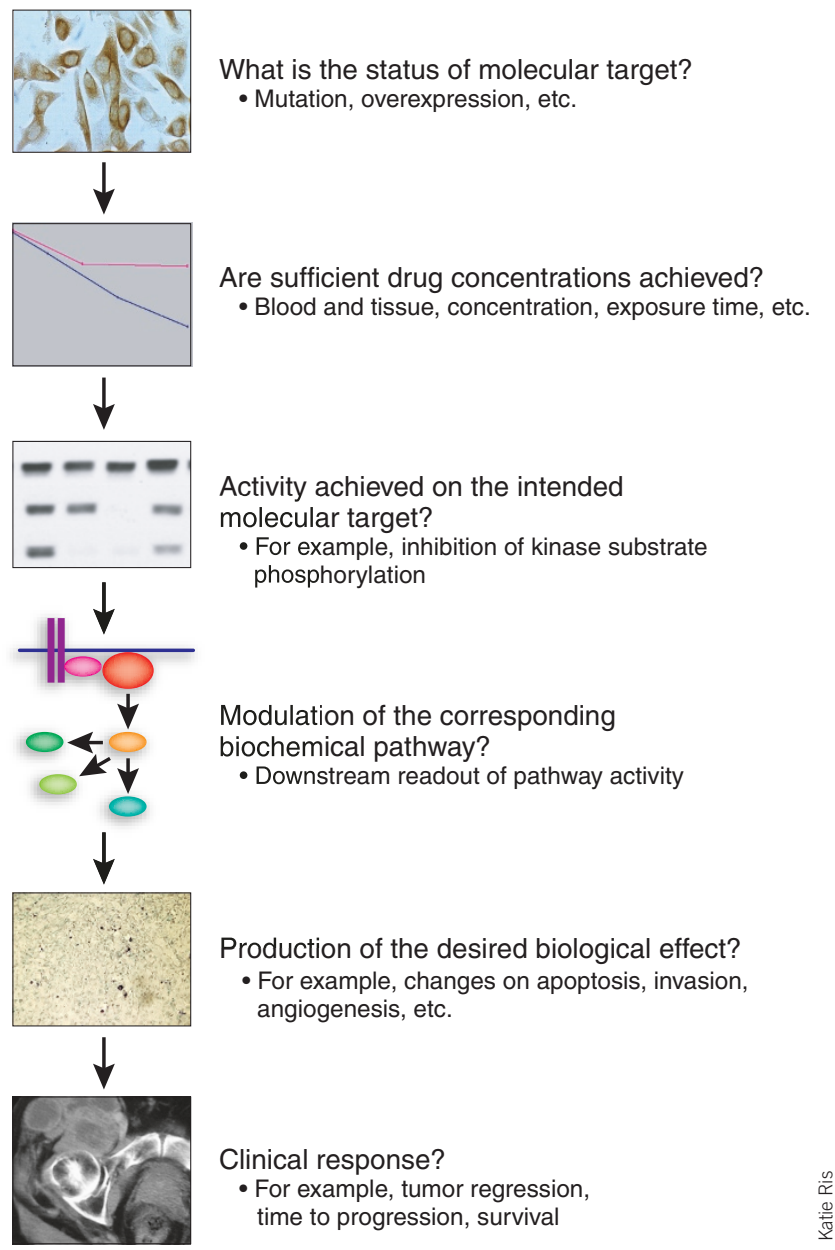

Figure 5 The pharmacologic audit trail. Rational drug discovery and development benefits from a clear understanding and measurement of a series of key parameters linking the status of the molecular target to the ultimate clinical activity. The measurement of these parameters can indicate proof of concept for target, pathway and biological effect modulation and provides the basis for selecting the optimal drug dose and schedule, and for go-no go decision making. Modified from refs. 7 and 112 with permission. Image for "Production of the desired biological effect?" provided courtesy of P. Hergenrother from Nat. Chem. Biol. 2, 543-550 (2006).

\section{Future prospects: toward bespoke cancer medicine}

So how far have we come- and where are we going - with small-molecule cancer therapeutics? We argue here that the glass is half full. There have been spectacular successes, led by Gleevec and Herceptin, that demonstrate proof of concept that major clinical benefit can be gained from targeting the driving oncogenic abnormalities responsible for particular types of cancer in particular subject populations. Will these drugs be a model to follow? In a way, yes, because the principle of the rational, targeted approach is now proven. But cancer is a formidably complex disease, and significant challenges have already been identified.

Many important cancer targets (for example, mutant p53, RAS and MYC) and oncogenic pathways (such as WNT) remain undrugged. Although drugging the cancer kinome is clearly achievable, many other target classes, notably phosphatases and most protein-protein interactions, have proved technically intractable so far. Nevertheless, success with MDM2 binding agents such as the nutlins ${ }^{117}$ and with BCL2 antagonists such as ABT-737 (ref. 118) shows that at least some proteinprotein interactions can be drugged. 
What else? The 95\% attrition rate for oncology drugs in the clinic is unacceptably high. We must prevent the expensive, late-stage casualties such as the matrix metalloprotease (MMP) inhibitors, the farnesyltransferase inhibitors and some receptor tyrosine kinase inhibitors. In the case of MMP inhibitors, there was a good rationale to develop these as anti-invasive agents and they did show activity in preclinical models. It is likely that they are casualties of being among the first of the new generation of molecularly targeted agents to enter the clinic, particularly in terms of their evaluation in late-stage disease rather than in earlierstage cancer, in which their activity may have been revealed more readily. However, it could be argued that the hurdle for therapeutic activity may be generally set too low and that we should be more rigorous in the level of preclinical activity required for entry into the clinic. On the other hand, given the uncertain predictiveness of our animal models ${ }^{99,100}$, it would be difficult at this time to set quantitative criteria for regression or cytostasis, particularly for first-in-class agents. But this is an area that requires close attention and highlights the need to integrate early clinical experience with ongoing preclinical drug discovery to reevaluate the optimum path into and through clinical development.

Cycle times in preclinical and clinical discovery and development generally need to be compressed. Also, it is clear that the age-old problem of drug resistance will still apply to the new molecular cancer therapeutics. Because of this and the multiple abnormalities contributing to very many cancers, a combinatorial therapeutic approach will be essential, as with cytotoxic cancer therapy and HIV-AIDS treatment.

The application and integration of new and existing techniques will shorten cycle times. High-throughput genomic, molecular and biochemical technologies will help to elucidate the complete range of mutational repertoires and hierarchies driving different cancers. This will help us in the molecular detection, classification, monitoring, treatment and, potentially, prevention of cancer. Both genetic and (increasingly) epigenetic changes will be important to understand. Better methods are needed to validate, select and prioritize new drug targets, including the use of high-throughput RNAi platforms. Understanding and exploiting oncogene addiction and other cancer dependencies will remain important. Greater emphasis on achieving synthetic lethal therapies (for example by exploiting DNA repair abnormalities, as with BRCA mutants) is justified ${ }^{10}$.

Emphasis so far has been on targets involved in cell cycle control and proliferation, and more recently in angiogenesis. The other hallmark traits of cancer need to be addressed more fully. Mechanism-based inducers of apoptosis are now entering the clinic. Telomerase inhibitors may be used to block immortalization. We need to revisit mechanism-based inhibition of invasion and evaluate this in more appropriate and careful trials than was the case for MMP inhibitors, particularly with regard to selection of subjects and stage of disease. Though it is an important goal, clinical evaluation of specifically targeted metastasis inhibitors is challenging because of the long timescales that may be required to obtain a meaningful end point.

In the broad sense, as stressed in this article, chemical biology methods will help to enhance the traditional linear process of drug development by providing means to interconnect the disparate stages. Lead generation will benefit from extracting more information from screening ${ }^{119}$ and from refined compound-selection criteria that take account of all the potential hurdles a drug must clear. Evaluation of efficacy, selectivity, ADMET, appropriate combinations and resistance liability for compounds at the earliest stages will provide a multidimensional SAR that will focus and speed up lead optimization, thereby providing rapid routes to the clinic. This may require further development of expert systems to correlate the very large amount of cheminformatic data generated as multiple properties are determined for increasing numbers of compounds ${ }^{120,121}$. Prototype small molecules and clinical agents will serve as tools to reinvestigate the underlying biological hypotheses, to discover modified or new strategies for targeted therapy, and to interrogate cancer models. Ongoing improvement of animal models ${ }^{122}$ and critical review of their clinical predictiveness is essential. Prospective analysis of the predictiveness of animal models may allow us to raise the preclinical hurdle for entry into the clinic.

Clinical feedback needs to be obtained more quickly, but also more intelligently. This can be done using better and more quantitative end points, and particularly by using molecular and imaging biomarkers. Clinical trial design needs to reflect the genetics and molecular cell biology of the target and the pharmacology of the drug; it should also reflect the expected outcome, which is often disease stabilization rather than rapid regression ${ }^{123}$. Biomarkers will be used increasingly to select people most likely to respond to a molecular cancer therapeutic, and to show proof of concept, monitor therapy and design optimal schedules. The pharmacologic audit trail can be used to aid decision making and manage risk.

Mechanisms of drug resistance can now be predicted and overcome by molecular and chemical biology techniques. Combinatorial multitarget inhibition will be essential in most cases. This can be achieved by using rationally selected combinations of highly targeted agents, according to the precise genetic and epigenetic makeup of the particular cancer, or by using intrinsic polypharmacy agents such as multitargeted kinase inhibitors or drugs affecting multiple downstream targets (for example, molecular chaperones or chromatin-modifying enzymes). Identifying the best drug combinations is difficult. There may be value in modulating the same target with different agents, hitting the same biological pathway at different levels, or inhibiting distinct pathways or hallmark traits simultaneously. Rational choice of effective combinations may be based on molecular knowledge. Use of chemical inhibitors alongside high-throughput RNAi technology can identify effective combinatorial targets ${ }^{11,28}$. Systematic HTS of drug pairs ${ }^{124}$ and systems-biology approaches are underway ${ }^{125}$. High-throughput inhibitor-selectivity profiling methods will be important for developing both highly selective and multitargeted agents 9 .

Much greater emphasis should be placed on understanding the complex signal transduction networks that are hijacked by malignant cells, rather than erroneously considering these as simple linear, textbook pathways. Better understanding of feedback and feedforward loops and of network robustness and sensitivity is needed. Mathematical models must be developed to predict signaling-network behavior and optimal points for intervention, and these must then be evaluated experimentally ${ }^{126}$.

An excellent example of the importance of feedback control was provided recently with the use of rapamycin-based mTOR inhibitors. mTOR is an important target for cancer therapy, but clinical results have been disappointing, except in renal cell cancer, in which the activity may be due to an antiangiogenic effect. Activity may be limited by a feedback loop involving the downstream ribosomal protein S6 kinase and the upstream adaptor insulin receptor substrate 1 (IRS1) ${ }^{127}$, thereby leading to activation of AKT. In a recent chemical biology approach in which a library of PI(3)K inhibitors was used to identify biological functions of various isoforms, it was shown that this problem can be overcome by the pyridofuropyrimidine inhibitor PI103, which simultaneously inhibits mTOR and the PI(3)K p110 $\alpha$, thereby causing a more complete blockade of the pathway and in particular preventing feedback activation ${ }^{6,128}$.

A concept of cancer biology that is likely to be important for targeted molecular therapeutics is that of tumor heterogeneity, and in particular the presence of tumor stem cells ${ }^{129}$. Such cells are by definition capable of repopulating the whole tumor, and therapies that are able to eradicate them will be critical.

We are in an exciting era in which there is great potential to develop rational, hypothesis-driven, mechanism-based molecular therapeutics 
for cancer. There are many challenges, but they can be addressed by the powerful techniques of genomics, molecular biology and chemical biology. The concept of a chemical probe for every protein encoded by the genome $\mathrm{e}^{130}$ can now be extended to the vision of achieving a molecularly targeted drug for all oncogenic proteins encoded by the cancer genome, or at least for every oncogenic pathway involved in the disease. There are excellent prospects for prolonging life and even curing people with cancer. A progressive advance toward the development of truly personalized cancer medicine can be predicted over the next five to ten years.

\section{ACKNOWLEDGMENTS}

This article is dedicated to our late friend and colleague F.T. (Tom) Boyle, who spent most of his successful career working on the medicinal chemistry of cancer drugs. The authors' work (http://www.icr.ac.uk/) is funded primarily by Cancer Research UK [CUK] Programme Grant C309/A2187, and P. Workman is a Cancer Research UK Life Fellow. We thank our many colleagues and collaborators for stimulating discussions.

\section{COMPETING INTERESTS STATEMENT}

The authors declare competing financial interests (see the Nature Chemical Biology website for details).

Published online at http://www.nature.com/naturechemicalbiology

Reprints and permissions information is available online at http://npg.nature.com/ reprintsandpermissions/

1. Kola, I. \& Landis, J. Can the pharmaceutical industry reduce attrition rates? Nat. Rev. Drug Discov. 3, 711-715 (2004).

2. Dalton, W.S. \& Friend, S.H. Cancer biomarkers-an invitation to the table. Science $\mathbf{3 1 2}$, 1165-1168 (2006)

3. Wesche, H., Xiao, S. \& Young, S.W. High-throughput screening for protein kinase inhibitors. Comb. Chem. High Throughput Screen. 8, 181-195 (2005).

4. Clemons, P.A. Complex phenotypic assays in high-throughput screening. Curr. Opin. Chem. Biol. 8, 334-338 (2004).

5. Noble, M.E.M., Endicott, J.A. \& Johnson, L.N. Protein kinase inhibitors: insights into drug design from structure. Science 303, 1800-1805 (2004).

6. Fan, Q.W. et al. A dual PI3 kinase/mTOR inhibitor reveals emergent efficacy in glioma. Cancer Cel/ 9, 341-349 (2006).

7. Workman, P. How much gets there and what does it do?: the need for better pharmacokinetic and pharmacodynamic endpoints in contemporary drug discovery and development. Curr. Pharm. Des. 9, 891-902 (2003).

8. Becker, F. et al. A three-hybrid approach to scanning the proteome for targets of small molecule kinase inhibitors. Chem. Biol. 11, 211-223 (2004).

9. Fabian, M.A. et al. A small molecule-kinase interaction map for clinical kinase inhibitors. Nat. Biotechnol. 23, 329-336 (2005).

10. Farmer, H. et al. Targeting the DNA repair defect in BRCA mutant cells as a therapeutic strategy. Nature 434, 917-921 (2005).

11. Morgan-Lappe, S. et al. RNAi-based screening of the human kinome identifies Aktcooperating kinases: a new approach to designing efficacious multi-targeted kinase inhibitors. Oncogene 25, 1340-1348 (2006).

12. Varmus, $H$. The new era in cancer research. Science 312, 1162-1165 (2006).

13. Workman, P. Drugging the cancer kinome: progress and challenges in developing personalized molecular cancer therapeutics. Cold Spring Harb. Symp. Quant. Biol. 70, 499-515 (2005).

14. Workman, P. Genomics and the second golden era of cancer drug development. Mol. Biosyst. 1, 17-26 (2005).

15. Marsham, P.R. et al. Design and synthesis of potent non-polyglutamatable quinazoline antifolate thymidylate synthase inhibitors. J. Med. Chem. 42, 3809-3820 (1999).

16. Vogelstein, B. \& Kinzler, K.W. Cancer genes and the pathways they control. Nat. Med. 10, 789-799 (2004).

17. Hanahan, D. \& Weinberg, R.A. The hallmarks of cancer. Cell 100, 57-70 (2000).

18. McDonald, E., Workman, P. \& Jones, K. Inhibitors of the HSP90 molecular chaperone: attacking the master regulator in cancer. Curr. Med. Chem. 6, 1091-1107 (2006).

19. Minucci, S. \& Pelicci, P.G. Histone deacetylase inhibitors and the promise of epigenetic (and more) treatments for cancer. Nat. Rev. Cancer 6, 38-51 (2006).

20. Capdeville, R., Buchdunger, E., Zimmerman, J. \& Matter, A. Glivec (ST571, imatinib), a rationally developed, targeted anticancer drug. Nat. Rev. Drug Discov. 1, 493-502 (2002).

21. Weinstein, I.B. Cancer. Addiction to oncogenes-the Achilles heal of cancer. Science 297, 63-64 (2002)

22. Benson, J.D. et al. Validating cancer drug targets. Nature 441, 451-456 (2006).

23. Solit, D. et al. BRAF mutation predicts sensitivity to MEK inhibition. Nature 439 , 358-362 (2006).

24. Garraway, L. et al. Integrative genomic analyses identify MITF as a lineage survival oncogene amplified in malignant melanoma. Nature 436, 117-122 (2005).

25. Futreal, P.A. et al. A census of human cancer genes. Nat. Rev. Cancer 4, 177-183 (2004).

26. Thomas, R.K. et al. Sensitive mutation detection in heterogeneous cancer specimens by massively parallel picoliter reactor sequencing. Nat. Med. 12, 852-855 (2006)
27. Davies, H. et al. Mutations of the BRAF gene in human cancer. Nature 417, 949-954 (2002).

28. Brummelkamp, T.R. et al. An shRNA barcode screen provides insight into cancer cell vulnerability to MDM2 inhibitors. Nat. Chem. Biol. 2, 202-206 (2006).

29. Chatterjee-Kishore, M. \& Miller, C.P. Exploring the sounds of silence: RNAi-mediated gene silencing for target identification and validation. Drug Discov. Today 10 $1559-1565$ (2005).

30. Lacouture, M.E. Mechanisms of cutaneous toxicities to EGFR inhibitors. Nat. Rev. Cancer 6, 803-812 (2006)

31. Newbatt, Y. et al. Identification of inhibitors of the kinase activity of oncogenic V600E BRAF in an enzyme cascade high-throughput screen. J. Biomol. Screen. 11, 145-154 (2006).

32. Park, S. et al. Hexachlorophene inhibits Wnt/beta-catenin pathway by promoting Siahmediated beta-catenin degradation. Mol. Pharmacol. 70, 960-966 (2006).

33. Hart, C.P. Finding the target after screening the phenotype. Drug Discov. Today 10, 513-519 (2005).

34. Luesch, $\mathrm{H}$. et al. A genome-wide overexpression screen in yeast for small-molecule target identification. Chem. Biol. 12, 55-63 (2005).

35. Evans, M.J., Saghatelian, A., Sorensen, E.J. \& Cravatt, B.F. Target discovery in smallmolecule cell-based screens by in situ proteome reactivity profiling. Nat. Biotechnol. 23, 1303-1307 (2005).

36. Lundholt, B.K. et al. Identification of Akt pathway inhibitors using redistribution screening on the FLIPR and the IN cell 3000 analyzer. J. Biomol. Screen. 10, 20-29 (2005).

37. Wolff, M. et al. Automated high content screening for phosphoinositide 3 kinase inhibition using an AKT 1 redistribution assay. Comb. Chem. High Throughput Screen. 9, 339-350 (2006).

38. Kau, T.R. et al. A chemical genetic screen identifies inhibitors of regulated nuclear export of a Forkhead transcription factor in PTEN-deficient tumor cells. Cancer Cell 4, 463-476 (2003).

39. Rees, D.C., Congreve, M., Murray, C.W. \& Carr, R.A.E. Fragment-based lead discovery. Nat. Rev. Drug Discov. 3, 660-672 (2004)

40. Gill, A.L. et al. Identification of novel p38alpha MAP kinase inhibitors using fragment based lead generation. J. Med. Chem. 48,414-426 (2005).

41. Card, G.L. et al. A family of phosphodiesterase inhibitors discovered by cocrystallography and scaffold-based drug design. Nat. Biotechnol. 23, 201-207 (2005).

42. Lipinski, C.A., Lombardo, F., Dominy, B.W. \& Feeney, P.J. Experimental and computational approaches to estimate solubility and permeability in drug discovery and development settings. Adv. Drug Deliv. Rev. 46, 3-26 (2001).

43. Lumley, J.A. Compound selection and filtering in library design. QSAR Comb. Sci. 24, 1066-1075 (2005).

44. Oprea, T.I., Davis, A.M., Teague, S.J. \& Leeson, P.D. Is there a difference between leads and drugs? A historical perspective. J. Chem. Inf. Comput. Sci.41, 1308-1315 (2001).

45. Veber, D.F., Johnson, S.R., Cheng, H., Ward, K.W. \& Kopple, K.D. Molecular properties that influence the oral bioavailability of drug candidates. J. Med. Chem. 45, 2615-2623 (2002).

46. Vieth, M. et al. Characteristic physical properties and structural fragments of marketed oral drugs. J. Med. Chem. 47, 224-232 (2004).

47. Lu, J.J. et al. Influence of molecular flexibility and polar surface area metrics on oral bioavailability in the rat. J. Med. Chem. 47, 6104-6107 (2004).

48. McGovern, S.L., Caselli, E., Grigorieff, N. \& Schoichet, B.K. A common mechanism underlying promiscuous inhibitors from virtual and high-throughput screening. J. Med. Chem. 45, 1712-1722 (2002).

49. Rishton, G.M. Nonleadlikeness and leadlikeness in biochemical screening. Drug Discov. Today 8, 86-96 (2003)

50. Kitchen, D.B., Decornez, H., Furr, J.R. \& Bajorath, J. Docking and scoring in virtual screening for drug discovery: methods and applications. Nat. Rev. Drug Discov. 3 935-949 (2004).

51. Lyne, P.D. et al. Identification of compounds with nanomolar binding affinity for checkpoint kinase-1 using knowledge-based virtual screening. J. Med. Chem. 47 1962-1968 (2004).

52. Muller, O. et al. Identification of potent Ras signaling inhibitors by pathway-selective phenotype-based screening. Angew. Chem. Int. Edn Engl. 43, 450-454 (2004).

53. Prien, 0 . Target-family-oriented focused libraries for kinases - conceptual design aspects and commercial availability. ChemBioChem 6, 500-505 (2005).

54. Muller, G. Medicinal chemistry of target family-directed masterkeys. Drug Discov. Today 8, 681-691 (2003).

55. Mann, J. Natural products in cancer chemotherapy: past, present and future. Nat. Rev. Cancer 2, 143-148 (2002)

56. Noren-Muller, A. et al. Discovery of protein phosphatase inhibitor classes by biologyoriented synthesis. Proc. Natl. Acad. Sci. USA 103, 10606-10611 (2006).

57. Tan, D.S. Diversity-oriented synthesis: exploring the intersections between chemistry and biology. Nat. Chem. Biol. 1, 74-84 (2005).

58. Clardy, J. \& Walsh, C. Lessons from natural molecules. Nature 432, 829-837 (2004).

59. Lipinski, C.A. \& Hopkins, A. Navigating chemical space for biology and medicine. Nature 432, 855-861 (2004).

60. Fry, D.C. \& Vassilev, L.T. Targeting protein-protein interactions for cancer therapy. J. Mol. Med. 83, 955-983 (2005)

61. Liu, Y. \& Gray, N.S. Rational design of inhibitors that bind to inactive kinase conformations. Nat. Chem. Biol. 2, 358-364 (2006).

62. Cohen, P. Protein kinases - the major drug targets of the twenty-first century? Nat. Rev. 
Drug Discov. 1, 309-315 (2002).

63. Ohren, J.F. et al. Structures of human MAP kinase kinase 1 (MEK1) and MEK2 describe novel noncompetitive kinase inhibition. Nat. Struct. Mol. Biol. 11, 1192-1197 (2004); erratum 12, 278 (2005)

64. Barnett, S.F., Bilodeau, M.T. \& Lindsley, C.W. The Akt/PKB family of protein kinases: a review of small molecule inhibitors and progress towards target validation. Curr. Top. Med. Chem. 5, 109-125 (2005).

65. Choi, J., Chen, J., Schreiber, S. \& Clardy, J. Structure of the FKBP12-rapamycin complex interacting with the binding domain of human FRAP. Science 273, 239-242 (1996).

66. Gorre, M.E. et al. Clinical resistance to STI-571 cancer therapy caused by BCR-ABL gene mutation or amplification. Science 293, 876-880 (2001).

67. Paez, J.G. et al. EGFR mutations in lung cancer: correlation with clinical response to gefitinib therapy. Science 304, 1497-1500 (2004)

68. Blencke, S., Ullrich, A. \& Daub, H. Mutation of threonine 766 in the epidermal growth factor receptor reveals a hotspot for resistance formation against selective tyrosine kinase inhibitors. J. Biol. Chem. 278, 15435-15440 (2003).

69. Shokat, K. \& Velleca, M. Novel chemical genetic approaches to the discovery of signal transduction inhibitors. Drug Discov. Today 7, 872-879 (2002).

70. Fry, D.W. Site-directed irreversible inhibitors of the erbB family of receptor tyrosine kinase as novel chemotherapeutic agents for cancer. Anticancer Drug Des. 15, 3-16 (2000).

71. Cohen, M.S., Zhang, C., Shokat, K.M. \& Taunton, J. Structural bioinformatics-based design of selective, irreversible kinase inhibitors. Science 308, 1318-1321 (2005).

72. Roe, S.M. et al. Structural basis for inhibition of of the Hsp90 molecular chaperone by the antitumour antibiotics radicicol and geldanamycin. J. Med. Chem. 42, 260-266 (1999).

73. Cheung, K.M. et al. The identification, synthesis, protein crystal structure and in vitro biochemical evaluation of a new 3,4-diarylpyrazole class of Hsp90 inhibitors. Bioorg. Med. Chem. Lett. 15, 3338-3343 (2005).

74. Chiosis, G. et al. A small molecule designed to bind to the adenine nucleotide pocket of Hsp90 causes Her2 degradation and the growth arrest and differentiation of breast cancer cells. Chem. Biol. 8, 289-299 (2001).

75. Wright, L. et al. Structure-activity relationships in purine-based inhibitor binding to HSP90 isoforms. Chem. Biol. 11, 775-785 (2004).

76. Finnin, M.S. et al. Structures of a histone deacetylase homologue bound to the TSA and SAHA inhibitors. Nature 401, 188-193 (1999).

77. Somoza, J.R. et al. Structural snapshots of human HDAC8 provide insights into the class I histone deacetylases. Structure 12, 1325-1334 (2004).

78. Hildmann, C. et al. Substrate and inhibitor specificity of class 1 and class 2 histone deacetylases. J. Biotechnol. 124, 258-270 (2006).

79. Koeller, K.M. et al. Chemical genetic modifier screens: small molecule trichostatin suppressors as probes of intracellular histone and tubulin acetylation. Chem. Biol. 10, 397-410 (2003)

80. Davis, A.M., Keeling, D.J., Steele, J., Tomkinson, N.P. \& Tinker, A.C. Components of successful lead generation. Curr. Top. Med. Chem. 5, 421-439 (2005).

81. Shuttleworth, S.J. et al. Design and synthesis of protein superfamily-targeted chemical libraries for lead identification and optimization. Curr. Med. Chem. 12, 1239-1281 (2005).

82. Lowinger, T.B., Riedl, B., Dumas, J. \& Smith, R.A. Design and discovery of small molecules targeting Raf-1 kinase. Curr. Pharm. Des. 8, 2269-2278 (2002).

83. Adams, J. et al. Potent and selective inhibitors of the proteasome: dipeptidyl boronic acids. Bioorg. Med. Chem. Lett. 8, 333-338 (1998).

84. Swinney, D.C. Biochemical mechanisms of drug action: what does it take for success? Nat. Rev. Drug Discov. 3, 801-808 (2004).

85. Hopkins, A.L., Groom, C.R. \& Alex, A. Ligand efficiency: a useful metric for lead selection. Drug Discov. Today 9, 430-431 (2004).

86. Knight, Z.A. \& Shokat, K.M. Features of selective kinase inhibitors. Chem. Biol. 12, 621-637 (2005)

87. Kung, C., Kenski, D.M., Krukenberg, K., Madhani, H.D. \& Shokat, K.M. Selective kinase inhibition by exploiting differential pathway selectivity. Chem. Biol. 13, 399407 (2006).

88. Bain, J., McLauchlan, H., Elliott, M. \& Cohen, P. The specificities of protein kinase inhibitors: an update. Biochem. J. 371, 199-204 (2003).

89. Brehmer, D. et al. Cellular targets of gefitinib. Cancer Res. 65, 379-382 (2005).

90. Wan, Y. et al. Synthesis and target identification of hymenialdisine analogues. Chem. Biol. 11, 247-259 (2004).

91. Obata, K., Sugano, K., Machida, M. \& Aso, Y. Biopharmaceutics classification by high throughput solubility assay and PAMPA. Drug Dev. Ind. Pharm. 30, 181-185 (2004).

92. Kerns, E.H. et al. Combined application of parallel artificial membrane permeability assay and Caco-2 permeability assays in drug discovery. J. Pharm. Sci. 93, 1440-1453 (2004).

93. Longley, D.B. \& Johnston, P.G. Molecular mechanisms of drug resistance. J. Pathol. 205, 275-292 (2005).

94. Nassar, A.E., Kamel, A.M. \& Clarimont, C. Improving the decision-making process in the structural modification of drug candidates: enhancing metabolic stability. Drug Discov. Today 9, 1020-1028 (2004).

95. Hutzler, J.M., Messing, D.M. \& Wienkers, L.C. Predicting drug-drug interactions in drug discovery: where are we now and where are we going? Curr. Opin. Drug Discov. Devel. 8, 51-58 (2005).

96. Raynaud, F.I. et al. Cassette dosing pharmacokinetics of a library of 2,6,9-trisubstituted purine cyclin-dependent kinase 2 inhibitors prepared by parallel synthesis. Mol. Cancer
Ther. 3, 353-362 (2004).

97. Smith, N.F. et al. Preclinical pharmacokinetics and metabolism of a novel diaryl pyrazole resorcinol series of heat shock protein 90 inhibitors. Mol. Cancer Ther. 5, 1628-1637 (2006).

98. Banerji, U. et al. Pharmacokinetic-pharmacodynamic relationships for the heat shock protein 90 molecular chaperone inhibitor 17-allylamino, 17-demethoxygeldanamycin in human ovarian cancer xenograft models. Clin. Cancer Res. 11, 7023-7032 (2005)

99. Sausville, E.A. \& Burger, A.M. Contributions of human tumour xenografts to anticancer drug development. Cancer Res. 66, 3351-3354 (2006).

100. Becher, O.J. \& Holland, E.C. Genetically engineered models have advantages over xenografts for preclinical studies. Cancer Res. 66, 3355-3359 (2006).

101. Whitebread, S., Hamon, J., Bojanic, D. \& Urban, L. In vitro safety pharmacology profiling: an essential tool for successful drug development. Drug Discov. Today 10, 1421-1433 (2005).

102. Haystead, T.A. The purinome, a complex mix of drug and toxicity targets. Curr. Top. Med. Chem. 6, 1117-1127 (2006)

103. Judson, I. Gastrointestinal stromal tumours (GIST): biology and treatment. Ann. Oncol. 13, 287-289 (2002)

104. Shah, N.P. et al. Overriding imatinib resistance with a novel ABL kinase inhibitor. Science 305, 399-401 (2004).

105. Strumberg, D. Preclinical and clinical development of the oral multikinase inhibitor sorafenib in cancer. Drugs Today (Barc). 41, 773-784 (2005).

106. Banerji, U. et al. Phase I pharmacokinetic and pharmacodynamic study of 17 -allylamino, 17-demethoxygeldanamycin in patients with advanced malignancies. J. Clin. Oncol. 23, 4152-4161 (2005).

107. Dymock, B.W. et al. Novel, potent small-molecule inhibitors of the molecular chaperone Hsp90 discovered through structure-based design. J. Med. Chem. 48, 4212-4215 (2005).

108. Kelly, W.K. \& Marks, P.A. Drug insight: histone deacetylase inhibitors-development of the new targeted anticancer agent suberoylanilide hydroxamic acid. Nat. Clin. Pract. Oncol. 2, 150-157 (2005).

109. Remiszewski, S.W. The discovery of NVP-LAQ824: from concept to clinic. Curr. Med. Chem. 10, 2393-2402 (2003).

110. Sawyers, C.L. Opportunities and challenges in the development of kinase inhibitor therapy for cancer. Genes Dev. 17, 2998-3010 (2003).

111. Frank, R. \& Hargreaves, R. Clinical biomarkers in drug discovery and development. Nat. Rev. Drug Discov. 2, 566-580 (2003).

112. Workman, P. Challenges of PK/PD measurements in modern drug development. Eur. J. Cancer 38, 2189-2193 (2002).

113. Workman, P. Auditing the pharmacological accounts for Hsp90 molecular chaperone inhibitors: unfolding the relationship between pharmacokinetics and pharmacodynamics. Mol. Cancer Ther. 2, 131-138 (2003).

114. Workman, P. \& Johnston, P.G. Genomic profiling of cancer: what next? J. Clin. Oncol. 23, 7253-7256 (2005).

115. Workman, P. et al. Minimally invasive pharmacokinetic and pharmacodynamic technologies in hypothesis-testing clinical trials of innovative therapies. J. Natl. Cancer Inst. 98, 580-598 (2006).

116. Garrett, M.D. et al. Novel isoquinoline-5-sulfonamides as biochemical and cellula inhibitors of PKB/AKt. Eur. J. Cancer Suppl. 2, 98 (2004).

117. Vassilev, L.T. et al. In vivo activation of the p53 pathway by small-molecule antagonists of MDM2. Science 303, 844-888 (2004).

118. Oltersdorf, T. et al. An inhibitor of $\mathrm{Bcl}-2$ family proteins induces regression of solid tumours. Nature 435, 677-681 (2005).

119. Inglese, J. et al. Quantitative high-throughput screening: a titration-based approach that efficiently identifies biological activities in large chemical libraries. Proc. Nat/. Acad. Sci. USA 103, 11473-11478 (2006).

120. Paolini, G.V. et al. Global mapping of pharmacological space. Nat. Biotechnol. 24, 805-815 (2006).

121. Shoemaker, R. The NCI60 human tumour cell line anticancer drug screen. Nat. Rev. Cancer 6, 813-823 (2006).

122. Sharpless, N.E. \& DePinho, R.A. The mighty mouse: genetically engineered mouse models in cancer drug development. Nat. Rev. Drug Discov. 5, 741-754 (2006).

123. Ratain, M.J. \& Eckardt, S.G. Phase II studies of modern drugs directed against new targets: if you are fazed, too, then resist RECIST. J. Clin. Oncol. 22, 4442-4445 (2004).

124. Borisy, A.A. et al. Systematic discovery of multicomponent therapeutics. Proc. Natl. Acad. Sci. USA 100, 7977-7982 (2003).

125. Fitzgerald, J.B., Schoeberl, B., Nielsen, U.B. \& Sorger, P.K. Systems biology and combination therapy in the quest for clinical efficacy. Nat. Chem. Biol. 2, 458-466 (2006).

126. Alves, R., Antunes, F. \& Salvador, A. Tools for kinetic modeling of biochemical networks. Nat. Biotechnol. 24, 667-672 (2006)

127. Harrington, L.S. et al. The TSC1-2 tumor suppressor controls insulin-PI3K signaling via regulation of IRS proteins. J. Cell Biol. 166, 213-223 (2004).

128. Workman P., Clarke, P.A., Guillard, S. \& Raynaud, F.I. Drugging the PI3 kinome. Nat. Biotechnol. 24, 794-796 (2006); corrigendum 24, 1033 (2006).

129. Clarke, M.F. \& Fuller, M. Stem cells and cancer: two faces of eve. Cel/ 124, 1111-1115 (2006).

130. Schreiber, S.L. Stuart Schreiber: biology from a chemist's perspective. Interview by Joanna Owens. Drug Discov. Today 9, 299-303 (2004).

131. Kassel, D.B. Applications of high-throughput ADME in drug discovery. Curr. Opin. Chem. Biol. 8, 339-345 (2004) 